\title{
Regular Cosmological Examples of Tree-Level Dilaton-Driven Models
}

\author{
Massimo Giovanninif \\ DAMTP, 21 Silver Street,Cambridge, CB3 9EW, United Kingdom
}

\begin{abstract}
We construct analytic solutions of the low energy (i.e. tree-level) string cosmological effective action. We work with the "minimal" field content (i.e. graviton and dilaton) in the absence of any dilaton potential and of any antisymmetric tensor field. Provided the metric is sufficiently inhomogeneous we obtain solutions whose curvature invariants are bounded and everywhere defined in time and space. The dilaton coupling and its associated energy density are regular and homogeneous. A phase of growing (and non-singular) dilaton coupling is compatible with the regularity of the curvature invariants without the addition of higher curvature (or higher genus) corrections to the tree-level effective action. We discuss the symmetries and the physical properties of the obtained solutions.
\end{abstract}

\footnotetext{
${ }^{1}$ e-mail: M.Giovannini@damtp.cam.ac.uk
} 


\section{Introduction and motivations}

A quite intriguing aspect of cosmological models based on the low energy string theory effective action is the occurrence of a phase of growing dilaton coupling that might trigger a number of interesting implications which were specifically addressed in the framework of the pre-big-bang inflationary models [1]. One of the distinctive aspects of this class of models is the amplification, via gravitational instability, of the vacuum inhomogeneities associated both with the metric and with the Abelian gauge fields [2]. If this picture is correct, we should live, at later times in an Universe filled with stochastic backgrounds of gravitational waves and of of Abelian gauge fields which, thanks to the magnetic flux conservation in a hot plasma, might also have interesting effects.

A lot of efforts have certainly been devoted to the analysis of the cosmological solutions of the low energy string theory effective action [3] in order to understand if the physical properties of those solutions were at all relevant for the explanation of some general features of our Universe. In all these studies one of the key assumptions was the complete homogeneity of the classical evolution of the geometry. It has been recently realized that it would be quite interesting to relax this very stringent assumption and, therefore, inhomogeneous string cosmological models were discussed from different points of view [4. 5 , but always in the framework of the low energy string theory effective action. There are of course different motivations for these investigations. In general, it is always quite dangerous to make strict assumptions about the form of the cosmological solutions. We deal here with solutions of a completely non-linear system of evolution equations and we cannot exclude, in principle, physical situations were the Universe was very inhomogeneous at early times evolving, ultimately, towards a homogeneous (and possibly isotropic) epoch at later times. In order to address these questions one should start, from the very beginning, with a completely inhomogeneous metric. Completely inhomogeneous configurations are also required in the analysis of the initial conditions of cosmological (inflationary) models. To relax the homogeneity assumption turned out to be relevant for understanding if (and when) inflation could take place starting from more generic initial conditions. An example in this direction are the investigations concerning the role played by initial (spherical) inhomogeneities in chaotic inflationary

models [6]. With the same motivations (but in the context of string inspired cosmological mod- 
els) quasi-homogenous metrics were used for the discussion of the initial value problem [5] in the pre-big-bang scenario [1, 7]. In this last class of models, the discussion of the initial conditions in a completely homogeneous metric can lead to quite different results [8]. We argue, therefore, that there is an urgent need of a better understanding of completely inhomogeneous configurations of the massless string modes.

We cannot a priori exclude that the features of inhomogeneous string cosmological models might be significantly different from the ones of their completely homogeneous counterpart.

An open question of (homogeneous) string cosmological models is the occurrence of curvature singularities [7] which turned out to be closely connected with the problem of a graceful exit from a phase of growing dilaton coupling [9], towards a phase of declining dilaton coupling. In a completely homogeneous metrics quite general (numerical) studies suggested that a phase of growing coupling is compatible with the regularity of the curvature invariants (in the String frame) provided higher order (curvature) corrections to the low energy (tree-level) effective action are included in the game [9]. This idea received recently new attention [10]. Moreover, the idea of a graceful exit transition from the initial phase of growing coupling to the subsequent standard radiation-dominated evolution has been the subject of many investigations [1].

In this paper we will not address the problem of the graceful exit but we will be concerned with the regularity of the curvature invariants.

In the case of a completely inhomogeneous metric is it still mandatory to hit a singularity after a phase of growing coupling? Is it possible to regularize the curvature invariants if the Universe was sufficiently inhomogeneous at early times?

These are the main question we will try to address and, in this sense, our approach is qualitatively different from the ones followed in Ref. [10, 11] (where homogeneity was assumed) and could be related to some pioneering attempts discussed in Ref. [12]. Regular (inhomogeneous) solutions of the low energy string theory effective action were constructed [12] by "boosting away" the curvature singularities through the $O(d, d)$ symmetries which were extensively studied in the past [13, 14] and which represent a natural generalization of the scale factor duality [1, 16]. The key observation was that starting with a generic singular cosmology in $1+1$ dimensions or with a $1+1$ dimensional black hole, the singularity gets "boosted away" by $O(2,2)$ transformation involving a third (originally flat) dimension. Moreover, by considering the inhomogeneous cosmo- 
logical model of Nappi and Witten [15] it was also shown that the singularities of the model can be "boosted away" by a $O(3,3)$ transformations involving a fifth dimension. The main conclusion of Ref. [12] was that, provided the coupling oscillates and provided an antisymmetric tensor background is included, the curvature invariants are regular. Therefore, there are no doubts that it is certainly physically relevant to relax the homogeneity assumption usually made in string inspired cosmological models. Following this logical line and applying the Occam razor we want to study inhomogeneous string cosmological models with the minimal field content (i.e. the gravi-dilaton action). In other words, we want to understand if it is possible to have simultaneously satisfied these requirements: growing coupling, regularity of the curvature invariants, absence of antisymmetric tensor field, absence of any dilaton potential. In this sense the aim of our investigation is different from (but complementary to) the one of Ref. [12].

To tackle this problem many different strategies can be certainly employed. The most general one would consist in trying to solve the low energy beta functions for a generic metric $g_{\mu \nu}(\vec{x}, t)$ depending simultaneously upon space and time. A simplification adopted in previous studies [5, 4] was also to stick to a diagonal inhomogeneous metric. Also the form of the inhomogeneous metric is, in a way, "minimal". In fact the simplest non-trivial case of fully inhomogeneous metrics is the one where the inhomogeneities are distributed along a line. In this case the whole spatial dependence is reduced to only one coordinate (which we took, in our examples, to run along the the $x$ axis). Needless to say that this is not the most general situation. At the same time there are no reasons to forbid this choice. A posteriori we finally found useful to impose an extra symmetry on the metric which will turn out to possess, in our discussion, two killing vector fields which are hypersurface orthogonal and orthogonal to each other. This form of the metric seems to be widely used in the study of inhomogeneous cosmological models in general relativity [17]. It is interesting to notice (as we will explicitly show) that this ansatz for the metric is compatible with a completely homogenous dilaton coupling which turns out to be, ultimately, only dependent upon time.

We solved exactly the low energy beta functions in the String frame by using all the assumptions listed above. We computed the curvature invariants associated with the obtained solutions and we found a class of regular (and parity-invariant) solutions where the curvature invariants are all bounded. These solutions describe the physical situation where a growing dilaton coupling 
smoothly evolves between two asymptotically constant regimes. The physical parameter describing the solutions is essentially the maximal energy density of the dilaton background in string units.

From a technical point of view, we have to mention that the most difficult part of our present exercise is to solve the off-diagonal component of the beta functions which do not contain second order derivatives with respect to time and which is then a constraint mixing first order time derivatives with first order spatial derivatives. Therefore, the first step will be the solution of the constraint. If the obtained solution will be compatible with the dilaton equation and with the remaining components of the beta functions, then, we will have a particular solution whose singularity properties can be investigated by the direct calculation of the curvature invariants.

Before closing this introduction we want to recall that the work presented in this paper is certainly overlapping with previous work performed in the context of general relativity. In particular in the limit of constant dilaton field our solutions match the well known inhomogeneous vacuum solutions of general relativity. The simplest inhomogeneous vacuum models are those with two space-like commuting killing vectors, known as orthogonally transitive $G_{2}$ cosmologies [18, 19. Moreover, useful background material for the present investigation can be also found in [20] where soliton solutions were discussed in space-times with two space-like Killing fields.

The plan of our paper is then the following. In Section 2 we will derive the explicit form of the evolution equations of the dilaton and of the metric in the String frame picture. In Section 3 we will briefly derive the dilaton vacuum solutions in the String frame and we will generalise them to the case of homogeneous dilaton background with inhomogeneous metric. In Section 4 we will focus our attention on the case of growing dilaton solutions and we will provide two physical examples whose main physical aspect is the parity invariance of the background geometry. Motivated the results of Section 4 we generalized our examples to a class of parity even solutions (Section 5) and we computed the curvature invariants in this situation. We found that they are regular as in the case of the examples of Section 4. Section 6 contains our concluding remarks. Because of the excessive length of the formulas contained in this paper we made the choice of reporting few (indeed relevant) technical results in the Appendix which then collects useful calculations for the interested reader. 


\section{Basic Equations}

The low energy string theory effective action can be written in the String frame and in the absence of antisymmetric tensor as [3]:

$$
S=-\frac{1}{\lambda_{s}^{2}} \int d^{4} x \sqrt{-g} e^{-\phi}\left[R+g^{\alpha \beta} \partial_{\alpha} \phi \partial_{\beta} \phi\right],
$$

where $\lambda_{s}$ is the string scale. We assume that the dilaton potential is completely negligible. We shall consider only the particular case of critical superstring theory with vanishing cosmological constant and six (frozen) internal dimensions. The evolution equations for the massless modes can be easily derived by varying the action with respect to the dilaton field and the metric:

$$
\begin{aligned}
& R-g^{\alpha \beta} \partial_{\alpha} \phi \partial_{\beta} \phi+2 \square \phi=0, \\
& R_{\mu \nu}-\frac{1}{2} g_{\mu \nu} R+\frac{1}{2} g_{\mu \nu} g^{\alpha \beta} \partial_{\alpha} \phi \partial_{\beta} \phi+\nabla_{\mu} \nabla_{\nu} \phi-g_{\mu \nu} \square \phi=0
\end{aligned}
$$

$\left(\nabla_{\alpha}\right.$ is the covariant derivative with respect to the metric $g_{\mu \nu} ; \nabla_{\alpha} \nabla_{\beta} \phi=\partial_{\alpha} \partial_{\beta} \phi-\Gamma_{\alpha \beta}^{\sigma} \partial_{\sigma} \phi ; \Gamma_{\alpha \beta}^{\sigma}$ are the Christoffel symbols). Using Eq. (2.2) into Eq. (2.3) we find the familiar form of the tree-level beta functions

$$
\begin{aligned}
& R-g^{\alpha \beta} \partial_{\alpha} \phi \partial_{\beta} \phi+2 \square \phi=0, \\
& R_{\mu}^{\nu}+\nabla_{\mu} \nabla^{\nu} \phi=0 .
\end{aligned}
$$

We now specialize to the case of a diagonal metric which admits two commuting space-like killing vector fields, both of which are hypersurface orthogonal 17]:

$$
g_{00}=A(x, t), \quad g_{x x}=-A(x, t), \quad g_{y y}=-B(x, t) C(x, t), \quad g_{z z}=-\frac{B(x, t)}{C(x, t)} .
$$

The corresponding line element is then

$$
d s^{2}=A(x, t)\left[d t^{2}-d x^{2}\right]-B(x, t)\left[C(x, t) d y^{2}+\frac{d z^{2}}{C(x, t)}\right] .
$$

From Eqs. (2.6) and (2.7) the Christoffel symbols, the Riemann tensors and the Weyl tensors can be easily computed. In Appendix A we report the Christoffel symbols, the Ricci tensors and the curvature scalar which are required in order to express directly Eqs. (2.4) and (2.5) in the metric (2.6). In the metric (2.6), Eqs (2.4) and (2.5) will produce a non-linear system of 
(partial) differential equations which we are going to solve. First of all suppose that the space-time dependence of the metric functions can be factorised:

$$
A(x, t)=a(t) \alpha(x), \quad B(x, t)=b(t) \beta(x), \quad C(x, t)=c(t) \kappa(x),
$$

(notice that the latin letters will denote the temporal part whereas the Greek ones will denote the spatial part of the metric functions). This decomposition has the great advantage, once inserted in Eqs. (2.4) and (2.5), of transforming the original system into a system of non-linear ordinary differential equations in the two variables $x$ and $t$. By inserting $\mathrm{Eq}(2.8)$ into the components of the Ricci tensors and into the curvature scalar given in Appendix A (see Eqs. (A.1)-(A.6)) we can write down explicitly Eq. (2.4) and all the components of Eq. (2.5) in the metric given in Eq. (2.6). The $(x x),(y y),(z z)$ and (00) components of Eq. (2.5) will be respectively:

$$
\begin{aligned}
& \frac{\ddot{a}}{a}+\frac{\dot{a}}{a} \frac{\dot{b}}{b}-\left(\frac{\dot{a}}{a}\right)^{2}-\frac{\dot{a}}{a} \dot{\phi}=2 \frac{\beta^{\prime \prime}}{\beta}+\left(\frac{\alpha^{\prime}}{\alpha}\right)^{\prime}+\left(\frac{\kappa^{\prime}}{\kappa}\right)^{2}-\left(\frac{\beta^{\prime}}{\beta}\right)^{2}-\frac{\alpha^{\prime}}{\alpha} \frac{\beta^{\prime}}{\beta} \quad(x x), \\
& \frac{\ddot{c}}{c}+\frac{\ddot{b}}{b}+\frac{\dot{b}}{b} \frac{\dot{c}}{c}-\left(\frac{\dot{c}}{c}\right)^{2}-\left(\frac{\dot{b}}{b}+\frac{\dot{c}}{c}\right) \dot{\phi}=\frac{\kappa^{\prime \prime}}{\kappa}+\frac{\beta^{\prime \prime}}{\beta}-\left(\frac{\kappa^{\prime}}{\kappa}\right)^{2}+\frac{\beta^{\prime}}{\beta} \frac{\kappa^{\prime}}{\kappa} \quad(y y), \\
& \frac{\ddot{b}}{b}+\left(\frac{\dot{c}}{c}\right)^{2}-\frac{\dot{b}}{b} \frac{\dot{c}}{c}-\frac{\ddot{c}}{c}-\left(\frac{\dot{b}}{b}-\frac{\dot{c}}{c}\right) \dot{\phi}=\frac{\beta^{\prime \prime}}{\beta}-\frac{\kappa^{\prime \prime}}{\kappa}+\left(\frac{\kappa^{\prime}}{\kappa}\right)^{2}-\frac{\beta^{\prime}}{\beta} \frac{\kappa^{\prime}}{\kappa} \\
& 2 \ddot{\phi}-\frac{\dot{a}}{a} \dot{\phi}+\left(\frac{\dot{a}}{a}\right)^{2}+\frac{\dot{a}}{a} \frac{\dot{b}}{b}+\left(\frac{\dot{b}}{b}\right)^{2}-\left(\frac{\dot{c}}{c}\right)^{2}-\frac{\ddot{a}}{a}-2 \frac{\ddot{b}}{b}=-\frac{\alpha^{\prime}}{\alpha} \frac{\beta^{\prime}}{\beta}-\left(\frac{\alpha^{\prime}}{\alpha}\right)^{\prime}
\end{aligned}
$$

whereas for Eq. (2.4) and for the mixed component (i.e. $(x 0))$ of Eq. (2.5) we will have respectively:

$$
\begin{aligned}
& 2 \ddot{\phi}+2 \frac{\dot{b}}{b} \dot{\phi}-\dot{\phi}^{2}+\frac{1}{2}\left(\frac{\dot{b}}{b}\right)^{2}-\frac{1}{2}\left(\frac{\dot{c}}{c}\right)^{2}-\frac{d}{d t}\left(\frac{\dot{a}}{a}\right)-2 \frac{\ddot{b}}{b}=\frac{1}{2}\left(\frac{\beta^{\prime}}{\beta}\right)^{2}-\frac{1}{2}\left(\frac{\kappa^{\prime}}{\kappa}\right)^{2} \\
& -\left(\frac{\alpha^{\prime}}{\alpha}\right)^{\prime}-2 \frac{\beta^{\prime \prime}}{\beta} \\
& \frac{\dot{b}}{b} \frac{\alpha^{\prime}}{\alpha}+\frac{\dot{a}}{a} \frac{\beta^{\prime}}{\beta}-\frac{\beta^{\prime}}{\beta} \frac{\dot{b}}{b}-\frac{\dot{c}}{c} \frac{\kappa^{\prime}}{\kappa}-\frac{\alpha^{\prime}}{\alpha} \dot{\phi}=0
\end{aligned}
$$

(in these equations and in all the paper we adopted the compact notation : ${ }^{\prime}=\frac{\partial}{\partial x}$ and $\cdot=\frac{\partial}{\partial t}$ ). In the system of Eqs. (2.9)-(2.13) at the left hand side of each equation we only have time-dependent quantities whereas at the right hand side we only have space-dependent quantities. The exception is represented by Eq. (2.14) which is not a dynamical equation but a constraint mixing spatial 
and temporal derivatives. The coupled system of non-linear differential equations is written in the case where the dilaton coupling is completely homogeneous, namely $\phi^{\prime}=0, \quad \phi(x, t) \equiv \phi(t)$. The solution of the system of coupled, non-linear differential equations derived in the previous equations will be the main goal of the next Section.

\section{Homogeneous dilaton solutions}

The first step in the derivation of our solutions with homogeneous dilaton will be to consider the constant dilaton case where $\dot{\phi}(t)=0$. In order to find a general expression able to solve the constraint, we sum and subtract Eqs. (2.10) and (2.11), obtaining, respectively,

$$
\frac{\ddot{b}}{b}=\frac{\beta^{\prime \prime}}{\beta}, \quad\left[\frac{d}{d t}\left(\frac{\dot{c}}{c}\right)+\frac{\dot{b}}{b} \frac{\dot{c}}{c}\right]=\left[\frac{d}{d x}\left(\frac{\kappa^{\prime}}{\kappa}\right)+\frac{\beta^{\prime}}{\beta} \frac{\kappa^{\prime}}{\kappa}\right] .
$$

A particular solution of Eq. (3.1) which might solve the constraint is

$$
b(t)=\cosh \mu t, \quad \beta(x)=\sinh \mu x .
$$

It is true that Eq. (3.1) can be solved also for another choices of trial functions: not all the possible choices are allowed by the off-diagonal constraint given in Eq. (2.14), which can now be re-written as

$$
\left[\mu \tanh \mu t\left(\frac{\alpha^{\prime}}{\alpha}\right)+\left(\frac{\dot{a}}{a}\right) \frac{\mu}{\tanh \mu x}-\mu^{2} \frac{\tanh \mu t}{\tanh \mu x}-\left(\frac{\dot{c}}{c}\right)\left(\frac{\kappa^{\prime}}{\kappa}\right)\right]=0
$$

Provided

$$
\frac{\dot{a}}{a}=c_{1} \tanh \mu t, \text { and } \quad \frac{\dot{c}}{c}=c_{2} \tanh \mu t,
$$

the time dependence appearing in Eq. (3.3) factorizes leading, ultimately, to a first-order (ordinary) differential relation involving only functions depending upon the spatial coordinates. Therefore direct (trivial) integration of Eqs. (3.1) and (3.3), together with the conditions (3.2) and (3.4), gives us the following first integrals

$$
\begin{aligned}
& \left(\frac{\kappa^{\prime}}{\kappa}\right)=\frac{c_{2}+c_{3}}{2}\left(\tanh \frac{\mu x}{2}\right)^{-1}+\frac{c_{2}-c_{3}}{2}\left(\tanh \frac{\mu x}{2}\right) \\
& \left(\frac{\alpha^{\prime}}{\alpha}\right)=\left[\left(\frac{c_{2}^{2}+c_{2} c_{3}-\mu c_{1}+\mu^{2}}{2 \mu}\right)\left(\tanh \frac{\mu x}{2}\right)^{-1}+\left(\frac{c_{2}^{2}-c_{2} c_{3}-\mu c_{1}+\mu^{2}}{2 \mu}\right) \tanh \frac{\mu x}{2}\right]
\end{aligned}
$$


where $c_{3}$ is now an integration constant and $c_{1}$ and $c_{2}$ are nothing but arbitrary constants which have to be fixed by requiring the compatibility of Eqs. (3.2) and (3.4) with the complete system given by by Eqs. (2.9)-(2.13). Let us therefore find the compatibility relations which have to be satisfied by $c_{1}, c_{2}$ and $c_{3}$. By direct substitution of Eqs. (3.2), (3.4), (3.5) and (3.6) into Eqs. (2.9) and (2.12) we get, respectively

$$
c_{3}^{2}=3 \mu^{2}+c_{2}^{2}-2 \mu c_{1}, \quad c_{2}^{2}=2 \mu c_{1}+\mu^{2} .
$$

Surprisingly enough the same two conditions also solve Eq. (2.13). Moreover Eqs. (2.10), (2.11) and (2.14) are automatically satisfied for every $c_{1}, c_{2}$ and $c_{3}$ not leading (as expected) to any further relation among the constants appearing in our trial solution. The present solutions are the String frame version of analogous inhomogeneous vacuum solutions previously discussed in the context of general relativity [17, 18].

After having solved the case where the dilaton was constant we can now discuss the case where the dilaton is time dependent. The ansatz given in Eqs. (3.2) and (3.4) can be slightly modified in order to match the time dependence of the dilaton field, which now appears, in the equations of motion (2.12)-(2.13) with its first and second derivatives. The idea is then to assume that in the presence of the dilaton field the solutions of our system of equations can be written as

$$
\left(\frac{\dot{a}}{a}\right)=c_{1} \tanh \mu t+\Gamma(t), \quad\left(\frac{\dot{b}}{b}\right)=\mu \tanh \mu t+\Delta(t), \quad\left(\frac{\dot{c}}{c}\right)=c_{2} \tanh \mu t+\Sigma(t)
$$

where, at the moment $\Gamma(t), \Delta(t)$ and $\Sigma(t)$, are three unknown functions which should be hopefully determined by solving explicitely the evolution equations of the metric and of the dilaton field. By comparing this ansatz with one made in the previous Section (see Eq. (3.4)) it is evident which kind of logic we followed since for $\Gamma=\Delta=\Sigma=0$ we exactly recover our Eq. (3.4). Notice that $\Gamma, \Delta$ and $\Sigma$ are only dependent upon time since we want to accommodate a homogenous dilaton .

With the ansatz (3.8) we then repeat step by step the procedure outlined in Section 1. First of all, we insert Eq. (3.8) into Eq. (2.14) and we get the condition which needs to be satisfied in order to solve the constraint:

$$
\left[\left(\frac{\alpha^{\prime}}{\alpha}\right)+\frac{\mu-c_{1}}{\tanh \mu x}\right]=\frac{c_{2}}{\mu}\left(\frac{\kappa^{\prime}}{\kappa}\right)-\frac{1}{\mu \tanh \mu t}\left\{\frac{\mu(\Gamma-\Delta)}{\tanh \mu x}-\left(\frac{\kappa^{\prime}}{\kappa}\right) \Sigma-\left(\frac{\alpha^{\prime}}{\alpha}\right) \dot{\phi}\right\} .
$$

By summing and subtracting Eqs. (2.10) and (2.11), we obtain

$$
\frac{\ddot{b}}{b}-\frac{\dot{b}}{b} \dot{\phi}=\frac{\beta^{\prime \prime}}{\beta}, \quad\left[\frac{d}{d t}\left(\frac{\dot{c}}{c}\right)-\frac{\dot{b}}{b} \frac{\dot{c}}{c}+\frac{\dot{c}}{c} \dot{\phi}\right]=\left[\frac{d}{d x}\left(\frac{\kappa^{\prime}}{\kappa}\right)+\frac{\beta^{\prime}}{\beta} \frac{\kappa^{\prime}}{\kappa}\right] .
$$


Inserting Eq. (3.8) into Eqs. (3.10), we find, respectively:

$$
\begin{aligned}
& \dot{\Delta}+[\Delta-\dot{\phi}] \Delta+\mu \tanh \mu t[2 \Delta-\dot{\phi}]=0, \\
& {\left[\frac{d}{d x}\left(\frac{\kappa^{\prime}}{\kappa}\right)+\frac{\beta^{\prime}}{\beta} \frac{\kappa^{\prime}}{\kappa}-c_{2} \mu\right]=\dot{\Sigma}+\tanh \mu t\left[\mu \Sigma+c_{2}(\Delta-\dot{\phi})\right]+\Sigma(\Delta-\dot{\phi}) .}
\end{aligned}
$$

Eqs. (3.9), (3.11) and (3.12) can be consistently solved if $\dot{\phi}(t)=\Delta(t)=\Gamma(t), \quad \Sigma=0$ leading to the solution

$$
\begin{aligned}
\left(\frac{k^{\prime}}{k}\right) & =\left[\frac{c_{3}+c_{2}}{2}\left(\tanh \frac{\mu x}{2}\right)^{-1}+\frac{c_{2}-c_{3}}{2} \tanh \frac{\mu x}{2}\right], \\
\left(\frac{\alpha^{\prime}}{\alpha}\right) & =\left[\frac{c_{2}^{2}+c_{2} c_{3}-\mu c_{1}+\mu^{2}}{2 \mu}\left(\tanh \frac{\mu x}{2}\right)^{-1}+\frac{c_{2}^{2}+c_{2} c_{3}-\mu c_{1}+\mu^{2}}{2 \mu} \tanh \frac{\mu x}{2}\right], \\
\Gamma(t) & =\frac{c_{4}}{\cosh \mu t},
\end{aligned}
$$

where $c_{3}$ and $c_{4}$ are integration constants. Substituting Eqs. (3.8) and (3.13) into Eq. (2.9) and Eq. (2.12) we obtain, respectively, the following compatibility conditions

$$
c_{3}^{2}=3 \mu^{2}+c_{2}^{2}-2 \mu c_{1} \geq 0, \quad c_{4}^{2}=c_{2}^{2}-\mu^{2}-2 \mu c_{1} \geq 0 .
$$

Finally going into Eq. (2.13) we deduce that

$$
2 c_{4}^{2}-\mu^{2}+6 \mu c_{1}=3 c_{2}^{2}-c_{3}^{2}
$$

which turns out to be noting but a trivial linear combination of the two conditions given in Eq. (3.14). As expected, Eqs. (2.10) and (2.11) are then satisfied without imposing any further condition on $c_{1}, c_{2}, c_{3}$ and $c_{4}$.

We are now ready to write down the explicit form of our solutions, namely:

$$
\begin{aligned}
A(x, t) & =e^{c_{4} g d(\mu t)}[\cosh \mu t]^{\frac{c_{1}}{\mu}}\left[\sinh \left(\frac{\mu x}{2}\right)\right]^{\frac{c_{2}^{2}+c_{2} c_{3}-\mu c_{1}+\mu^{2}}{\mu^{2}}}\left[\cosh \left(\frac{\mu x}{2}\right)\right]^{\frac{c_{2}^{2}-c_{2} c_{3}-\mu c_{1}+\mu^{2}}{\mu^{2}}}, \\
B(x, t) & =e^{c_{4} g d(\mu t)} \cosh \mu t \sinh \mu x \\
C(x, t) & =[\cosh \mu t]^{\frac{c_{2}}{\mu}}\left[\sinh \left(\frac{\mu x}{2}\right)\right]^{\frac{c_{2}+c_{3}}{\mu}}\left[\cosh \left(\frac{\mu x}{2}\right)\right]^{\frac{c_{2}-c_{3}}{\mu}}, \\
\phi(t) & =\frac{c_{4}}{\mu} g d(\mu t)-c_{5},
\end{aligned}
$$


where $g d(\mu t)=\arctan [\sinh \mu t]$ is the "Gudermannian" [21] or hyperbolic amplitude. Notice that the limit $c_{4} \rightarrow 0$ is slightly ill defined since, from Eq. (3.14), $c_{2}$ might take imaginary values and the inequalites are, in this limit, strict equalities. Therefore, the general expression of the solution (3.16) also changes, in this limit.

Since the hyperbolic amplitude always grows, the dilaton will either increase or decrease depending upon the sign of $c_{4}$. Therefore, the physical meaning of $c_{5}$ is connected with the initially small (perturbative) value of the coupling constant, whereas $c_{4}$ is connected with its "kinetic" energy. The physical situation we are describing with this solutions is really a regime of very small dilaton coupling and in this sense we will fine-tune $c_{5}$ to a quite small value. In this way higher

loop (genus) corrections will be automatically subleading. This procedure is very reminiscent of what happens in the pre-big-bang scenario [1] where a (quite long) small coupling regime emerges naturally from the tree-level (Kasner-like) solutions. In the pre-big-bang case, the Kasner-like solutions have two "branches" separated by a (curvature) singularity, and then, for each growing coupling solution there is also a decreasing coupling solution. We notice that, also in this sense, our solutions have close analogies to the pre-big-bang case since, depending upon the value of $c_{4}$ there are two branches: one of increasing coupling $\left(c_{4}>0\right)$ and the other of decreasing coupling $\left(c_{4}<0\right)$. In the pre-big-bang case the singularity cannot be removed (at tree-level). We will show, in the next two sections, that in our solutions the two branches are not analytically connected but, at the same time, the curvature invariants, the dilaton kinetic energy are always well defined and regular for any $x, t$ at least for a family of solutions contained in Eq. (3.16) (see Sections 4 and 5 for a discussion of this point).

\section{Two physical examples}

From Eq. (3.16) each particular solution of the system of Eqs. (2.4) and (2.5) can be specified by fixing $c_{1}$ and $c_{2}$ and by computing $c_{3}$ and $c_{4}$ from Eqs. (3.14). We tried different possible sets of parameters and we found that not all the choices of $c_{1}$ and $c_{2}$ will necessarily lead to the regularity of the curvature invariants. The class of regular solutions seems to be connected to the behaviour of the metric tensor under (discrete) parity transformations (see the following Section). At the same time we point out that there are choices of $c_{1}$ and $c_{2}$ which lead to completely regular 
curvature invariants. In this Section we discuss then particular examples of solutions contained in Eq. (3.16) that share this quite interesting property. In order to do that we will be inspired by some physical requirement, namely we would like to have regularity of the curvature invariants and, simultaneously, growing dilaton coupling which corresponds to $\dot{\phi}>0$.

For the set of parameters given by

$$
c_{1}=6 \mu, \quad c_{2}=5 \mu, \quad c_{3}=-4 \mu, \quad c_{4}=2 \sqrt{3} \mu,
$$

Eqs. (3.14) are clearly solved. The explicit form of the solution of Eq. (3.16) can then be written, using Eqs. (4.1), as:

$$
\begin{aligned}
& A(x, t)=e^{2 \sqrt{3} g d(\mu t)}[\cosh \mu t]^{6}\left[\cosh \left(\frac{\mu x}{2}\right)\right]^{40}, \quad B(x, t)=e^{2 \sqrt{3} g d(\mu t)} \cosh \mu t \sinh \mu x \\
& C(x, t)=[\cosh \mu t]^{5}\left[\sinh \left(\frac{\mu x}{2}\right)\right]\left[\cosh \left(\frac{\mu x}{2}\right)\right]^{9}, \quad \phi(t)=2 \sqrt{3} g d(\mu t)-c_{5} .
\end{aligned}
$$

The dilaton is an increasing function whose maximal value is given by $\phi(+\infty)=\sqrt{3} \pi-c_{5}$ (recall that $\lim _{t \rightarrow-\infty} g d(\mu t)=-(3 / 2) \pi$ and that $\lim _{t \rightarrow+\infty} g d(\mu t)=(\pi / 2)$ ). Now, our discussion is based on the (tree-level) action given by Eq. (2.1) which is valid provided $g(\phi) \equiv \exp [\phi / 2]=$ $\exp \left[\frac{c_{4}}{2} g d(\mu t)-c_{5}\right] \ll 1$. If this is not the case, higher genus corrections should be included. Consequently, for the compatibility of the solutions discussed in the present Section with the effective description adopted in Section 2 we have to require that the dilaton starts its time evolution deep in its perturbative regime and this can be achieved by fixing $c_{5}$. In the example given by the solution (4.2) we have that $g(+\infty)=\exp \left(\sqrt{3} \pi-c_{5}\right) \ll 1$, which implies that $c_{5} \gg \sqrt{3} \pi$. Therefore choosing, for instance $c_{5} \sim 10 \sqrt{3} \pi$ we get that $g(-\infty)=\exp (-13 \sqrt{3} \pi)$ and $g(+\infty)=\exp (-9 \sqrt{3} \pi)$.

Consider now a further example, namely

$$
c_{1}=5 \mu, \quad c_{2}=4 \mu, c_{3}=-3 \mu, \quad, \quad c_{4}=\sqrt{5} \mu .
$$

Using Eqs. (4.3) into Eq. (3.16) we get

$$
\begin{aligned}
& A(x, t)=e^{\sqrt{5} g d(\mu t)}[\cosh \mu t]^{5}\left[\cosh \left(\frac{\mu x}{2}\right)\right]^{24}, \quad B(x, t)=e^{\sqrt{5} g d(\mu t)} \cosh \mu t \sinh \mu x, \\
& C(x, t)=[\cosh \mu t]^{4}\left[\sinh \left(\frac{\mu x}{2}\right)\right]\left[\cosh \left(\frac{\mu x}{2}\right)\right]^{7}, \quad \phi(t)=\sqrt{5} g d(\mu t)-c_{5} .
\end{aligned}
$$


The calculation of the curvature invariants for the solutions (4.2) and (4.4) can be now performed. In order to summarise our results we can say that each of the curvature invariants can be written (in a generalised notation) as:

$$
\mathcal{I}=\mu^{4} f(x, t)[\cosh \mu t]^{-a}\left[\cosh \frac{\mu x}{2}\right]^{-b}
$$

$(\mathcal{I}$ labels a generic curvature invariant; $f(x, t)$ is a regular expression containing combinations of hyperbolic functions and it changes for each specific invariant [see Appendix B]; $a$ and $b$ also change depending upon the invariant under consideration, [see also Table 1]).

From Eq. (4.5) and from the various forms of $f(x, t)$ which can be deduced from Appendix B, we notice that $\mathcal{I}$ does not have any pole for any finite value od $x$ and $t$. Moreover, we point out that for large $x$ and $t$ the curvature invariants are suppressed. The magnitude of the suppression depends upon $a$ and $b$ which are reported in Table 1 but can also be read-off directly from Eqs. (B.1)-(B.4). The only nasty thing which could then occur is that for some (large) value of $x$ and/or $t$ some of the invariants explode because $f(x, t)$ grows much faster than the suppression factor, parameterised, for each invariant, by $a$ and $b$. Keeping $x=x_{c}$ (where $x_{c}$ is some finite value of $x$ ) and leaving $t$ to run we find that $f\left(x_{c}, t\right) \sim[\cosh \mu t]^{4}$ for large $t$. Vice-versa keeping $t$ frozen at $t_{c}$ (where $t_{c}$ is some finite value of $t$ ) we find that (in the worse case) $f\left(x, t_{c}\right) \sim\left[\cosh \frac{\mu x}{2}\right]^{4}$. Finally, by varying $x$ and $t$ simultaneously we see that $f(x, t)$ can grow, at most like $\left[\cosh \frac{\mu x}{2}\right]^{2}[\cosh \mu t]^{4}$ or like $\left[\cosh \frac{\mu x}{2}\right]^{4}[\cosh \mu t]^{2}$. Therefore we see that the growth of $f(x, t)$ is always suppressed by the high (negative) powers (i.e. $a$ and $b$ ) of the hyperbolic cosinus appearing in Eq. (4.5). We want now to make few more physical comments concerning the validity of the present solutions. The only dimension-full parameter appearing in the solution (4.2) and (4.4) is $\mu=1 / L$ where $L$ is, for the moment, a length scale which completely specifies each solution which describes the "spreading" of the curvature invariants around the origin (i.e. $x=0, t=0$ ). The only fundamental scale appearing in Eq. (2.1) is the string scale $\lambda_{s}$. Correspondingly there is also a typical energy connected with $\lambda_{s}$ which is the string energy, namely $E_{s}=1 / \lambda_{s}$. The effective action (2.1) which was the starting point of our considerations holds for energies $E \ll E_{s}$. In fact for $E \sim E_{s}$ higher order corrections in the string tension $\alpha^{\prime}=\lambda_{s}^{-2}$ should be included. In order to be consistent with our approach we have to assume that the typical scale $L$ has to be large compared to the string scale and this means that $L>\lambda_{s}$. In the opposite case (i.e. $\left.L<\lambda_{s}\right), \lambda_{s}$ 


\begin{tabular}{|c|c|c|c|c|}
\hline$c_{4}=2 \sqrt{3} \mu$ & $R_{\mu \nu \alpha \beta} R^{\mu \nu \alpha \beta}$ & $C_{\mu \nu \alpha \beta} C^{\mu \nu \alpha \beta}$ & $R_{\alpha \beta} R^{\alpha \beta}$ & $R^{2}$ \\
\hline$a$ & 16 & 16 & 16 & 16 \\
\hline$b$ & 84 & 84 & 82 & 80 \\
\hline
\end{tabular}

\begin{tabular}{|c|c|c|c|c|}
\hline$c_{4}=\sqrt{5} \mu$ & $R_{\mu \nu \alpha \beta} R^{\mu \nu \alpha \beta}$ & $C_{\mu \nu \alpha \beta} C^{\mu \nu \alpha \beta}$ & $R_{\alpha \beta} R^{\alpha \beta}$ & $R^{2}$ \\
\hline$a$ & 14 & 14 & 14 & 14 \\
\hline$b$ & 52 & 52 & 50 & 48 \\
\hline
\end{tabular}

Table 1: We summarize the exponents of the suppression factor appearing in Eq. (4.5). The upper part of the table refers to the case of Eq. (4.2), whereas the lower part of the table refers to the case of Eq. (4.4). The explicit analytical results leading to this table can be found in the Appendix B, by setting, respectively, $\alpha \equiv c_{4} / \mu=2 \sqrt{3}$ and $\alpha=c_{4} / \mu \sqrt{5}$ in Eqs. (B.1. (B.4).

would not turn out as the minimal length scale. Therefore if $L>\lambda_{s}$, we will also have $\mathcal{I}<\lambda_{s}^{-4}$. In Fig. 11 the scalar curvature (squared) is reported for the two solutions given in Eqs. (4.2) and (4.4) when $L=10 \lambda_{s}$. The corresponding analytical results are reported in Eq. (B.4) choosing, respectively $\alpha=2 \sqrt{3}$ and $\alpha=\sqrt{5}$. From these analytical expressions we can clearly see that by tuning $L=1 / \mu$ for length scales larger than $\lambda_{s}, R^{2}$ decreases (in string units) and its spreading around the origin increases. We can also look at the behaviour of the time derivative of the dilaton which is illustrated ( Fig. 2) and of the related coupling constant (Fig. 3).

The solutions derived in Section 3 and the examples reported in the present Section can be described either in the String frame or in the Einstein frame [23]. In the String frame the fundamental parameter of the theory is the String length $\lambda_{s}$. Notice that in this frame the String scale is truly a constant, whereas the Planck scale evolves according to a relation which can be parameterised (at low energy and low coupling) as $\lambda_{P}(t)=e^{\frac{\phi}{2}} \lambda_{s}$. The Einstein frame is defined by the conformal transformation which diagonalizes the gravitational action by decoupling the dilaton from the Einstein-Hilbert Lagrangian.In the Einstein frame $\lambda_{P} \sim 10^{-33} \mathrm{~cm}$ is constant whereas the String scale evolves according to $\lambda_{s}(t)=e^{-\frac{\Phi}{2}} \lambda_{P}$ (where $\Phi$ is the Einstein frame dilaton). The String frame and Einstein frame dilatons are simply related and, moreover, in four dimensions they are indeed equal, whereas the String frame $\left(g_{\mu \nu}\right)$ and Einstein frame $\left(G_{\mu \nu}\right)$ metrics are connected 


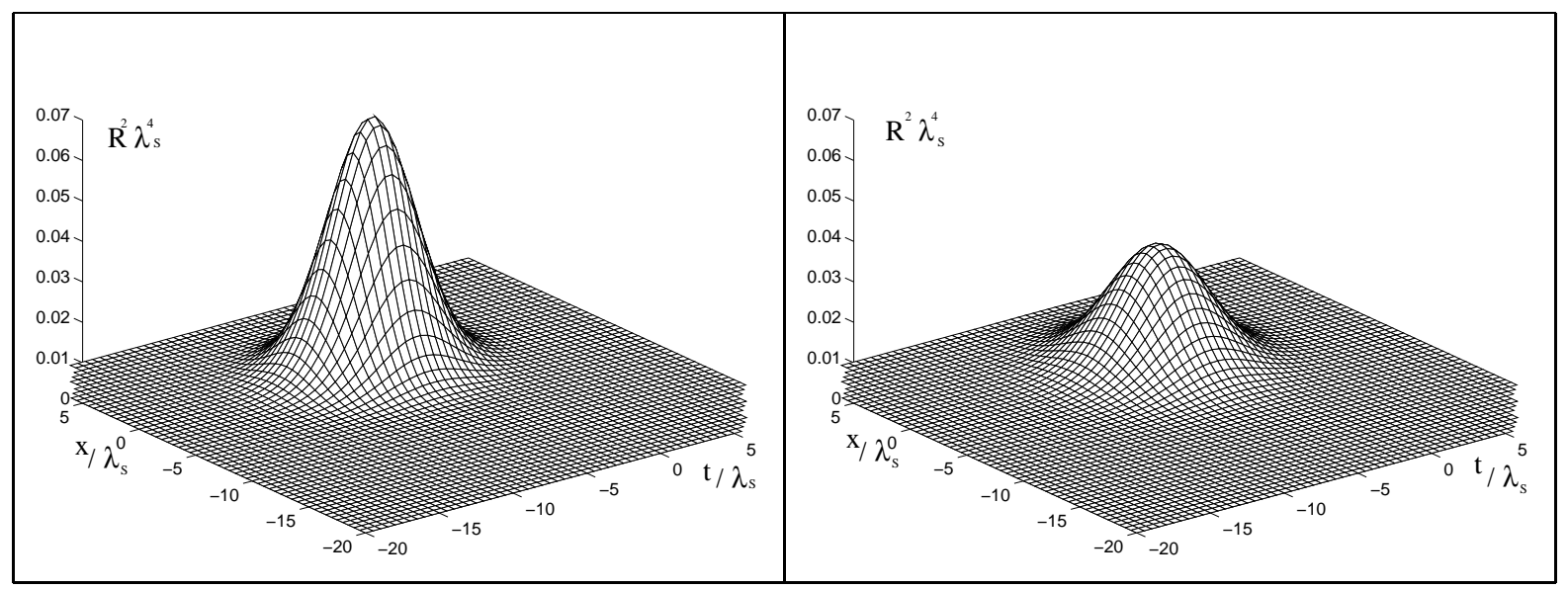

Figure 1: We plot the $R^{2}$ invariants corresponding to the solution (4.2) (left) and to the solution (4.4) (right). The analytic form of these invariants is given in Appendix B (Eq. (B.4)) by choosing $\alpha=2 \sqrt{3}$ (for the plot at the left) and $\alpha=\sqrt{5}$ (for the plot at the right). Here we also took $L=10 \lambda_{s}$
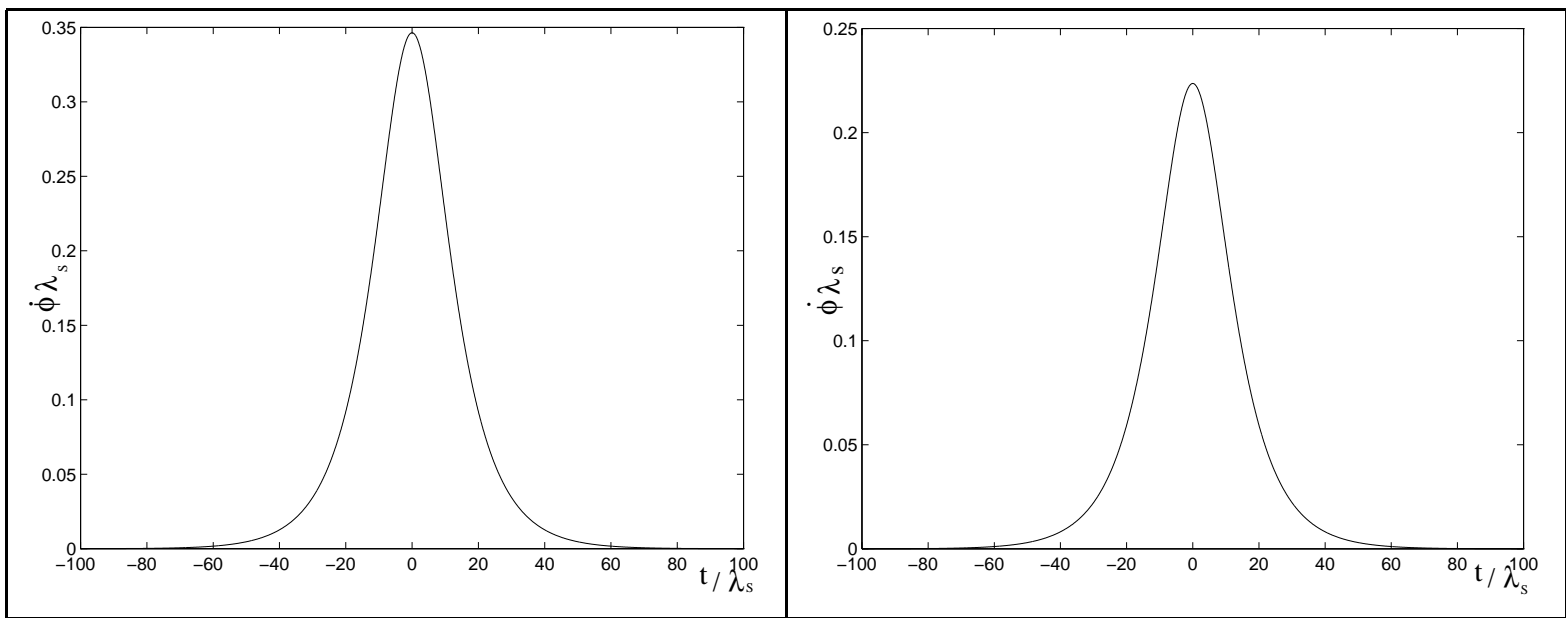

Figure 2: We plot the dilaton kinetic term for the two exact solutions discussed in this Section. The left figure corresponds to the case given in Eq. (4.2), whereas the right one corresponds to Eq. (4.4). We notice the absence of any singularity. 


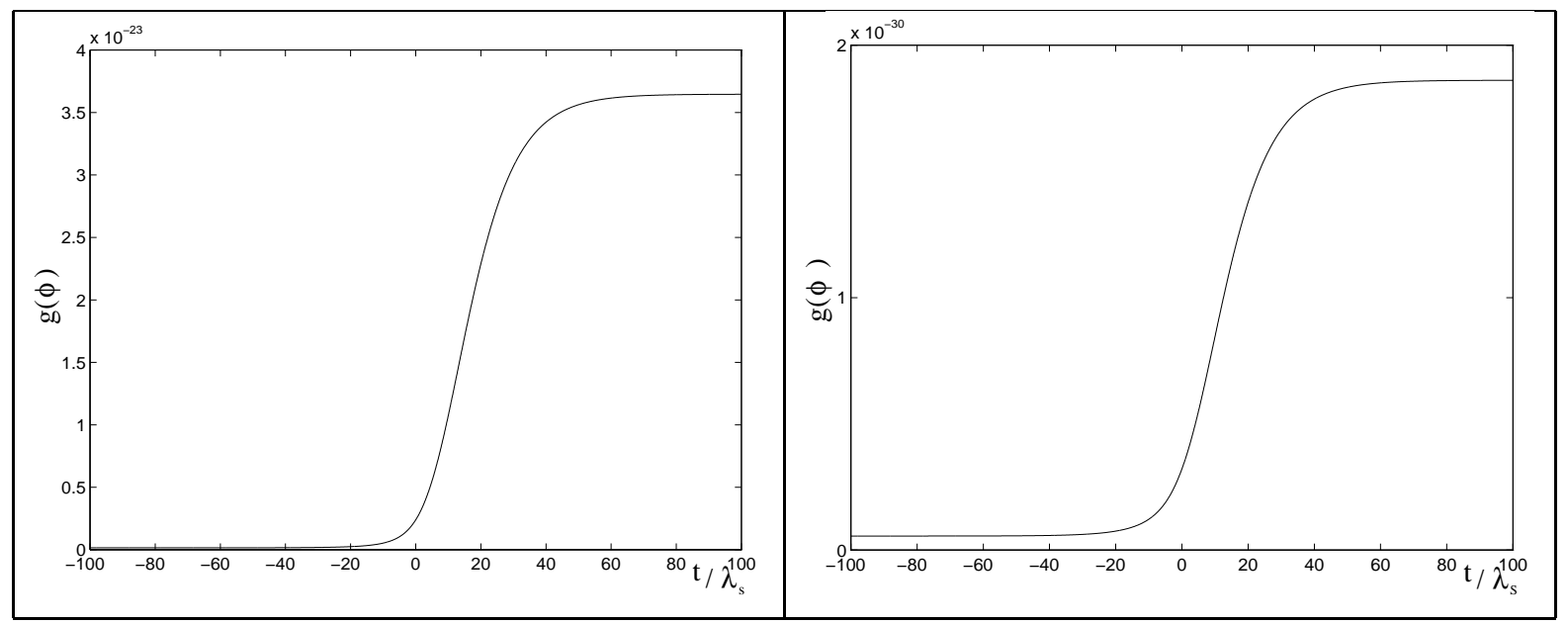

Figure 3: We report the evolution of the dilaton coupling in the solutions given by Eq. (4.2) (left) and by Eq. (4.4) (right). We took $c_{5}=10 \sqrt{3} \pi$ (left) and $c_{5}=10 \sqrt{5} \pi$ (right). Provided the dilaton coupling starts its evolution deep in the perturbative regime, $g(\phi) \ll 1$ for every time.

by a conformal rescaling involving the dilaton coupling $\left(g_{\mu \nu}=e^{\Phi} G_{\mu \nu}\right)$.

We transformed our solutions in the Einstein frame and we investigated the emerging picture which can be summarized by saying that the curvature invariants are also regular and everywhere defined. At this point, we would be tempted to call the solutions we just discussed "singularityfree". In order to name a generic solution of the low energy String effective action "singularityfree" we should discuss the geodesic completeness of the metric both in the Einstein and in the String frame pictures. From the point of view of the interpretation of our solutions, moreover, the geodesic completeness in the String frame seems even more crucial, since strings moving in curved space follow geodesics in the String frame but not in the Einstein frame [24]. To show that the geodesics do not hit a boundary in both frames is anyway a quite long computational task. We prefer to postpone it to further investigations mainly because the calculations reported here are already quite heavy. We do not have, at the moment, any argument either favouring or disfavouring the geodesic completeness and we believe that this can only come out from a precise calculation.

There is a second very important issue. The Einstein frame picture of our solutions involves an energy momentum tensor which does not violate any of the energy conditions which are part of 
the hypotheses of the powerful Hawking-Penrose (HP) theorems [25, 26]. Therefore the question which naturally arises is which other hypothesis of the HP theorems is violated. There are indeed examples of truly non-singular (inhomogeneous) solutions in general relativity which have an energy-momentum tensor of a perfect fluid [27]. In that context it was rigourously proven that the so-called initial or boundary condition of the HP theorems was the one failing to hold. This would mean, in our context, that we have to fine-tune (as we had!) the initial conditions quite heavily in order not to bump into a singularity. This point is indeed closely connected with the behaviour of the geodesics and with their completeness and therefore it certainly requires further studies. The same considerations should be performed in the String frame which is perhaps the most useful for the physical intuition (as it was noticed in the case of the pre-big-bang scenario [1]). We are not aware, at the moment, of any general singularity theorem in this case and therefore we are not in condition of making any kind of general statement going beyond the solutions we just presented.

\section{$5 \quad$ Parity invariant solutions with growing coupling}

The two physical examples presented in the previous Section share a quite amusing physical property, namely the invariance of the line element corresponding to each one of the solutions given in Eqs. (4.2) and (4.4) under discrete parity transformations. In fact by computing, from Eq. (2.7), the line element associated to the examples reported in Eq. (4.2) and (4.4) we find that it is invariant under the transformation $x \rightarrow-x$. Surprisingly enough, we will show that parity invariant metrics lead to regular curvature invariants. This will allow us to define a class of regular, inhomogeneous models with growing coupling.

We start by noting that, from the structure of the metric written in Eqs. (3.16) the diagonal elements of the metric tensor given in Eq. (2.6) are even under parity transformations provided the spatial $(x)$ dependence appears in functions which are themselves even under parity. Given the form of the metric (3.16) we see that, in order to preserve parity, the spatial dependence has to occur either through powers of the hyperbolic cosinus (which is parity even) or through even powers of the hyperbolic sinus. We then impose the following two extra conditions to the 
parameters $c_{1}, c_{2},, c_{3}, c_{4}$ specifying the solutions (3.16):

$$
c_{2}^{2}+c_{2} c_{3}-\mu c_{1}+\mu^{2}=0, \quad c_{2}+c_{3}=\mu
$$

In Eqs. (3.16) the first condition of Eq. (5.1) sets to zero the power of the hyperbolic sinus appearing in $A(x, t)$ whereas the second condition sets to one the power of the hyperbolic cosinus appearing in $C(x, t)$. Notice that with this choices the components of the metric are all even under $x \rightarrow-x$. Therefore, the full system of conditions obeyed by the solution given in Eq. (3.16) is

$$
\begin{aligned}
& \left(\frac{c_{3}}{\mu}\right)^{2}=3+\left(\frac{c_{2}}{\mu}\right)^{2}-2\left(\frac{c_{1}}{\mu}\right), \\
& \left(\frac{c_{4}}{\mu}\right)^{2}=\left(\frac{c_{2}}{\mu}\right)^{2}-2\left(\frac{c_{1}}{\mu}\right)-1, \\
& \left(\frac{c_{2}}{\mu}\right)^{2}=\left(\frac{c_{1}}{\mu}\right)-\left(\frac{c_{2}}{\mu}\right)\left(\frac{c_{3}}{\mu}\right), \\
& \left(\frac{c_{3}}{\mu}\right)+\left(\frac{c_{2}}{\mu}\right)=1 .
\end{aligned}
$$

Now calling, for simplicity $\alpha=c_{4} / \mu, \gamma=c_{2} / \mu, \delta=c_{1} / \mu$ and and eliminating $c_{3} / \mu$ through Eq. (5.2) from the previous equations we obtain the following system:

$$
\begin{aligned}
& \gamma+1=\delta, \\
& \alpha^{2}=\gamma^{2}-1-2 \delta, \\
& 2 \gamma+2=2 \delta .
\end{aligned}
$$

Eqs. (5.6) and (5.8) are clearly not independent and, therefore, the solution given in Eq. (3.16) can be expressed only in terms of one (physical) parameter which we choose to be $\alpha$. This turns out to be a physically interesting parametrization since $\mu^{2} \alpha^{2}=\dot{\phi}^{2}(0)$. In this sense $|\alpha|$ estimates the (maximal) energy density of the dilaton background in string units. The sign of $\alpha$ automatically selects solutions either with growing coupling or with decreasing coupling. Thus, using Eqs. (5.5), (5.6) and (5.7), we have that the metric of Eq. (3.16) can be re-written as

$$
\begin{aligned}
A(x, t) & =e^{ \pm \alpha g d(\mu t)}[\cosh \mu t]^{2 \pm \sqrt{\alpha^{2}+4}}\left[\cosh \left(\frac{\mu x}{2}\right)\right]^{2 \sqrt{\alpha^{2}+4}\left[\sqrt{\alpha^{2}+4} \pm 1\right]}, \\
B(x, t) & =e^{ \pm \alpha g d(\mu t)} \cosh \mu t \sinh \mu x \\
C(x, t) & =[\cosh \mu t]^{1 \pm \sqrt{\alpha^{2}+4}}\left[\sinh \left(\frac{\mu x}{2}\right)\right]\left[\cosh \left(\frac{\mu x}{2}\right)\right]^{1 \pm 2 \sqrt{\alpha^{2}+4}}, \\
\phi(t) & = \pm \alpha g d(\mu t)-\beta .
\end{aligned}
$$


(the plus and minus signs correspond, respectively, to solutions with growing and decreasing dilaton coupling). In Appendix B we computed the curvature invariants in the case of growing coupling solutions. We indeed found that the requirement of parity invariance of the solutions seem to be winning since the curvature invariants are regular. Notice that the curvature invariants of the two previous examples (reported in Section 4) can be derived from the general result of this Section by setting, respectively, $\alpha=2 \sqrt{3}$ and $\alpha=\sqrt{5}$. Also in the case of the class of solutions discussed in the present Section the curvature invariants vanish asymptotically as can be argued from the results reported in Appendix B. The curvature invariants can be also computed in the case of decreasing dilaton solution. We did this exercice and we found, again regular solutions. From the more mathematical results reported in this Section (and from the two examples of Section 4 illustrating the physical properties of our solutions) we see that something quite unexpected happens, namely the fact that the regularity of the curvature invariants can already be achieved at tree level (without any curvature correction). Moreover, as shown in Section 4 for two particular cases, we can set initial conditions in such a way that the coupling constant is always much smaller than one. We notice that these results are not in contrast with previous discussions [9] suggesting that the only way of regularizing the curvature invariants was to invoke higher curvature corrections. In fact those studies were always postulating a completely homogeneous (and often isotropic) four dimensional geometry. The physical picture emerging from our detailed analytical calculations is that the inhomogeneities are quite an essential ingredient in order to regularize the curvature invariants. On a purely physical ground, moreover, it does not seem unreasonable to have strong inhomogeneities at very small scales. As we noted in the introduction our results confirm and extend previous ideas concerning possible avoidance of the singularities in completely inhomogenous string cosmological models [12. In agreement with [12 we showed that regular solutions are possible at tree-level. At the same time we showed that this conclusion holds also in the presence of a growing coupling solution and in the absence of any extra field. We believe that our new results should be taken as a serious motivation in order to perform a fully consistent numerical study of inhomogeneous string cosmological models. These studies might extend (and perhaps change) some of the conclusions drawn in the completely homogeneous case [9]. In particular, numerical studies might clarify if it is at all possible to find inhomogeneous examples evolving at late times towards a completely homogeneous state. 
In fact a quite unphysical feature shared by our models and by the ones of [12 is their eternal inhomogeneity. It is certainly tue [17] that the homogeneous limit of a inhomogeneous model is already quite delicate in general relativity. In spite of the possible subtleties we can say that a good measure of how inhomogeneous and anisotropic is a solution at late times can be the ratio

of the Weyl invariant $\left(C^{\mu \nu \alpha \beta} C_{\mu \nu \alpha \beta}\right)$ over the Riemann invariant $\left(R^{\mu \nu \alpha \beta} R_{\mu \nu \alpha \beta}\right)$. In our case, from the results of Appendix B, this quantity goes to a constant at for $t \rightarrow+\infty$. A similar behaviour can be found in [12]. Numerical studies could also give us some clue concerning the role played by the compactification radii which we took frozen but which should be included in the game of inhomogeneous models. Up to now this possibility was never considered.

\section{Concluding remarks}

In this paper we gave some examples of regular dilaton driven solutions of the low energy beta functions without including any dilaton potential, any higher $\alpha^{\prime}$ (curvature) corrections, any higher genus corrections, any antisymmetric tensor field any dilaton potential. We took the six compactification radii to be constant and we looked at some special class of inhomogeneous metric. We showed that if the metric is sufficiently inhomogeneous the curvature invariants are bounded for every $x$ and $t$. The only definite conclusion that we feel like stating is that regular (but inhomogeneous) solutions of the evolution equations of the dilaton and of the geometry are more common (at tree-level) than in the completely homogenous case where no-go theorems probably apply. Since in our examples the coupling is always very small and the curvature invariants always smaller than one (in string units) the tree-level treatment seems, a posteriori, not meaningless. The curvature invariants (including the Weyl invariants) vanish, asymptotically for large times. Moreover the metric is invariant under parity transformations.

We leave to future studies many unanswered questions. Can we promote the regular solutions we spotted to true singularity free solutions? If this is the case are the geodesics complete in the Einstein frame? Which hypotheses of the singularity theorems turn out to be violated? Can we formulate the singularity theorems (in a consistent way) directly in the String frame or we have always to go to the Einstein frame? Should we consider regular only those solutions which are bounded in both frames? 


\section{Acknowledgments}

The author would like to thank M. Gasperini and G. Veneziano for a careful reading of the draft and for very useful comments. The author wishes also to thank former discussions with H. Soleng.

\section{APPENDIX}

\section{A Christoffel symbols and Ricci tensors}

In this Appendix we will give the general formulas for the Christoffel symbols and Ricci tensors computed in the case of the diagonal metric given in Eq. (2.6) with two commuting space-like killing vectors which are hypersurface orthogonal.

The Christoffel symbols can be easily derived from the metric (2.6) and they are reported in Table 2, whereas the Ricci tensors and the curvature scalar can be obtained, after some algebra,

once the Christoffel symbols are known. Our conventions for the Ricci tensor will be $R_{\mu \nu}=R_{\alpha \mu \nu}{ }^{\alpha}$ (where $R_{\mu \nu \alpha}{ }^{\beta}=\partial_{\mu} \Gamma_{\nu \alpha}{ }^{\beta}-\ldots$ is the Riemann tensor). The $(x x),(y y),(z z)$ and (00) components of the Ricci tensors are, respectively

$$
\begin{aligned}
& R_{x}^{x}=-\frac{1}{2 A}\left\{-\left(\frac{\dot{A}}{A}\right)^{2}+\frac{\dot{A}}{A} \frac{\dot{B}}{B}+\frac{\ddot{A}}{A}+\left(\frac{A^{\prime}}{A}\right)^{2}+\frac{A^{\prime}}{A} \frac{B^{\prime}}{B}+\left(\frac{B^{\prime}}{B}\right)^{2}-\left(\frac{C^{\prime}}{C}\right)^{2}-\frac{A^{\prime \prime}}{A}-2 \frac{B^{\prime \prime}}{B}\right\}, \\
& R_{y}^{y}=-\frac{1}{2 A}\left\{\frac{\dot{B}}{B} \frac{\dot{C}}{C}-\left(\frac{\dot{C}}{C}\right)^{2}+\frac{\ddot{B}}{B}+\frac{\ddot{C}}{C}-\frac{B^{\prime}}{B} \frac{C^{\prime}}{C}+\left(\frac{C^{\prime}}{C}\right)^{2}-\frac{B^{\prime \prime}}{B}-\frac{C^{\prime \prime}}{C}\right\}, \\
& R_{z}^{z}=-\frac{1}{2 A}\left\{\left(\frac{\dot{C}}{C}\right)^{2}-\frac{\ddot{B}}{B} \frac{\dot{C}}{C}+\frac{\ddot{B}}{B}-\frac{\ddot{C}}{C}+\frac{B^{\prime}}{B} \frac{C^{\prime}}{C}-\left(\frac{C^{\prime}}{C}\right)^{2}-\frac{B^{\prime \prime}}{B}+\frac{C^{\prime \prime}}{C}\right\} \\
& R_{0}^{0}=\frac{1}{2 A}\left\{\left(\frac{\dot{A}}{A}\right)^{2}+\frac{\dot{A}}{A} \frac{\dot{B}}{B}+\left(\frac{\dot{B}}{B}\right)^{2}-\left(\frac{\dot{C}}{C}\right)^{2}-\frac{\ddot{A}}{A}-2 \frac{\ddot{B}}{B}-\left(\frac{A^{\prime}}{A}\right)^{2}+\frac{A^{\prime}}{A} \frac{B^{\prime}}{B}+\frac{A^{\prime \prime}}{A}\right\}
\end{aligned}
$$

The $(0 x)$ component and the curvature scalar will be instead:

$$
\begin{gathered}
R_{x}^{0}=\frac{1}{2 A}\left\{\frac{\dot{B}}{B} \frac{A^{\prime}}{A}+\frac{\dot{A}}{A} \frac{B^{\prime}}{B}+\frac{B^{\prime}}{B} \frac{\dot{B}}{B}-\frac{\dot{C}}{C} \frac{C^{\prime}}{C}-2 \frac{\dot{B}^{\prime}}{B}\right\}, \\
R \equiv R_{\alpha}^{\alpha}=\frac{1}{2 A}\left\{2\left(\frac{\dot{A}}{A}\right)^{2}+\left(\frac{\dot{B}}{B}\right)^{2}-\left(\frac{\dot{C}}{C}\right)^{2}-2 \frac{\ddot{A}}{A}-4 \frac{\ddot{B}}{B}-2\left(\frac{A^{\prime}}{A}\right)^{2}-\left(\frac{B^{\prime}}{B}\right)^{2}+\left(\frac{C^{\prime}}{C}\right)^{2}+2 \frac{A^{\prime \prime}}{A}+4 \frac{B^{\prime \prime}}{B}\right\} .
\end{gathered}
$$




\begin{tabular}{|c|c|}
\hline$\Gamma_{x x}^{x}=\frac{1}{2} \frac{\partial \log A}{\partial x}$ & $\Gamma_{y y}^{x}=-\frac{C B}{2} \frac{\partial \log (B C)}{\partial x}$ \\
\hline$\Gamma_{z z}^{x}=-\frac{B}{2 A C} \frac{\partial \log (B C)}{\partial x}$ & $\Gamma_{x 0}^{x}=\frac{1}{2} \frac{\partial \log A}{\partial t}$ \\
\hline$\Gamma_{00}^{x}=\frac{1}{2} \frac{\partial \log A}{\partial x}$ & $\Gamma_{x y}^{y}=\frac{1}{2} \frac{\log (B C)}{\partial x}$ \\
\hline$\Gamma_{y 0}^{y}=\frac{1}{2} \frac{\log (B C)}{\partial t}$ & $\Gamma_{x z}^{z}=\frac{1}{2} \frac{\partial \log (B / C)}{\partial x}$ \\
\hline$\Gamma_{z 0}^{z}=\frac{1}{2} \frac{\partial \log (B / C)}{\partial t}$ & $\Gamma_{x x}^{0}=\frac{1}{2} \frac{\partial \log A}{\partial t}$ \\
\hline$\Gamma_{y y}^{0}=\frac{C B}{2 A} \frac{\partial \log (C B)}{\partial t}$ & $\Gamma_{z z}^{0}=\frac{B}{2 A C} \frac{\partial \log (B / C)}{\partial t}$ \\
\hline$\Gamma_{x 0}^{0}=\frac{1}{2} \frac{\partial \log A}{\partial x}$ & $\Gamma_{00}^{0}=\frac{1}{2} \frac{\partial \log A}{\partial t}$ \\
\hline
\end{tabular}

Table 2: We report the Christoffel symbols computed from the metric given in Eq. (2.6).

\section{B Curvature invariants for growing dilaton solutions}

From the (parity invariant) class of solutions given in Eq. (5.9) we can compute the curvature invariants, namely curvature scalar together with the squares of the Riemann, Weyl and Ricci tensors. The result is the following

$$
\begin{aligned}
R_{\mu \nu \alpha \beta} R^{\mu \nu \alpha \beta} & =\frac{\mu^{4}}{32} e^{-2 \alpha g d(\mu t)}[\cosh \mu t]^{-8-2 \sqrt{\alpha^{2}+4}}\left[\cosh \frac{\mu x}{2}\right]^{-4-4 \sqrt{\alpha^{2}+4}\left(1+\sqrt{\alpha^{2}+1}\right)}\left\{s_{1}(\alpha)\right. \\
& +s_{2}(\alpha) \cosh 2 \mu t+s_{3}(\alpha) \cosh 4 \mu t+s_{4}(\alpha) \cosh \mu x+s_{5}(\alpha) \cosh 2 \mu x \\
& +s_{6}(\alpha) \cosh 2 \mu t \cosh 2 \mu x+s_{7}(\alpha) \cosh 2 \mu t \cosh \mu x \\
& +s_{8}(\alpha) \cosh 4 \mu t \cosh \mu x+s_{9}(\alpha) \sinh \mu t+s_{10}(\alpha) \sinh \mu t \cosh 2 \mu x \\
& \left.+s_{11}(\alpha) \sinh \mu t \cosh \mu x\right\}, \\
C_{\mu \nu \alpha \beta} C^{\mu \nu \alpha \beta} & =\frac{\mu^{4}}{96} e^{-2 \alpha g d(\mu t)}[\cosh \mu t]^{-8-2 \sqrt{\alpha^{2}+4}}\left[\cosh \frac{\mu x}{2}\right]^{-4-4 \sqrt{\alpha^{2}+4}\left(1+\sqrt{\alpha^{2}+4}\right)}\left\{w_{1}(\alpha)\right. \\
& +w_{2}(\alpha) \cosh 4 \mu t+w_{4}(\alpha) \cosh \mu x+w_{5}(\alpha) \cosh 2 \mu x \\
& +w_{6}(\alpha) \cosh 2 \mu t \cosh 2 \mu x+w_{7}(\alpha) \cosh 2 \mu t \cosh \mu x \\
& \left.+w_{8}(\alpha) \cosh 4 \mu t \cosh \mu x\right\}, \\
R_{\alpha \beta} R^{\alpha \beta} & =\frac{\alpha^{2} \mu^{4}}{16} e^{-2 \alpha g d(\mu t)}[\cosh \mu t]^{-8-2 \sqrt{\alpha^{2}+4}}\left[\cosh \frac{\mu x}{2}\right]^{-2-4 \sqrt{\alpha^{2}+4}\left(1+\sqrt{\alpha^{2}+4}\right)}\left\{r_{1}(\alpha)\right. \\
& +r_{2}(\alpha) \cosh 2 \mu t+r_{3}(\alpha) \cosh \mu x+r_{4}(\alpha) \cosh 2 \mu t \cosh \mu x
\end{aligned}
$$




$$
\begin{aligned}
& \left.+r_{5}(\alpha) \sinh \mu t+r_{6}(\alpha) \sinh \mu t \cosh \mu x\right\} \\
R^{2} & =\alpha^{4} \mu^{4} e^{-2 \alpha g d(\mu t)}[\cosh \mu t]^{-8-2 \sqrt{\alpha^{2}+4}}\left[\cosh \frac{\mu x}{2}\right]^{-4 \sqrt{\alpha^{2}+4}\left(1+\sqrt{\alpha^{2}+4}\right)}
\end{aligned}
$$

The $\alpha$-dependent coefficients appearing in our expressions are reported in Table 3. Notice that the two examples specifically discussed in Section 4 arise by setting, respectively, $\alpha=2 \sqrt{3}$ and $\alpha=\sqrt{5}$. Plugging these values in Eqs. (B.1)-(B.4) we get the corresponding curvature invariants. We want to mention that we did the calculations of the curvature invariants also in the Einstein frame after having transformed the solutions according to the general conventions (discussed in Section 4) which relate the two frames. The curvature invariants turned out to be regular also in the Einstein frame. 


\begin{tabular}{|c|c|}
\hline$s_{1}(\alpha)$ & $1152+416 \alpha^{2}+824 \alpha^{4}+6 \alpha^{6}+\sqrt{\alpha^{2}+4}\left(504+98 \alpha^{2}+14 \alpha^{4}\right)$ \\
\hline$s_{2}(\alpha)$ & $384+536 \alpha^{2}+124 \alpha^{4}+8 \alpha^{6}+\sqrt{\alpha^{2}+4}\left(96+164 \alpha^{2}+20 \alpha^{4}\right)$ \\
\hline$s_{3}(\alpha)$ & $384+216 \alpha^{2}+38 \alpha^{4}+2 \alpha^{6}+\sqrt{\alpha^{2}+4}\left(168+66 \alpha^{2}+6 \alpha^{4}\right)$ \\
\hline$s_{4}(\alpha)$ & $48-108 \alpha^{2}-78 \alpha^{4}-6 \alpha^{6}-\sqrt{\alpha^{2}+4}\left(24+190 \alpha^{2}+18 \alpha^{4}\right)$ \\
\hline$s_{5}(\alpha)$ & $192+104 \alpha^{2}+16 \alpha^{4}+\sqrt{\alpha^{2}+4}\left(192+36 \alpha^{2}+4 \alpha^{4}\right)$ \\
\hline$s_{6}(\alpha)$ & $72 \alpha^{2}+12 \alpha^{4}+\sqrt{\alpha^{2}+4}\left(36 \alpha^{2}+4 \alpha^{4}\right)$ \\
\hline$s_{7}(\alpha)$ & $\left.-1728-688 \alpha^{2}-120 \alpha^{4}-8 \alpha^{6}-\left(864+232 \alpha^{2}+24 \alpha^{4}\right) \sqrt{\alpha^{2}+4}\right)$ \\
\hline$s_{8}(\alpha)$ & $-144-108 \alpha^{2}-18 \alpha^{4}-\left(72+42 \alpha^{2}+6 \alpha^{4}\right) \sqrt{\alpha^{2}+4}$ \\
\hline$s_{9}(\alpha)$ & $96 \alpha^{3}+24 \alpha^{3} \sqrt{\alpha^{2}+4}$ \\
\hline$s_{10}(\alpha)$ & $32 \alpha^{3}+8 \alpha^{3} \sqrt{\alpha^{2}+4}$ \\
\hline$s_{11}(\alpha)$ & $128 \alpha^{3}+32 \alpha^{3} \sqrt{\alpha^{2}+4}$ \\
\hline$w_{1}(\alpha)$ & $3456+1488 \alpha^{2}+216 \alpha^{4}+12 \alpha^{6}+\sqrt{\alpha^{2}+4}\left(1512+390 \alpha^{2}+30 \alpha^{4}\right)$ \\
\hline$w_{2}(\alpha)$ & $1152+1128 \alpha^{2}+282 \alpha^{4}+18 \alpha^{6}+\sqrt{\alpha^{2}+4}\left(288+300 \alpha^{2}+48 \alpha^{4}\right)$ \\
\hline$w_{3}(\alpha)$ & $1152+648 \alpha^{2}+114 \alpha^{4}+6 \alpha^{6}+\sqrt{\alpha^{2}+4}\left(504+198 \alpha^{2}+18 \alpha^{4}\right)$ \\
\hline$w_{4}(\alpha)$ & $-144-492 \alpha^{2}-266 \alpha^{4}-18 \alpha^{6}-\left(72+378 \alpha^{2}+54 \alpha^{4}\right) \sqrt{\alpha^{2}+4}$ \\
\hline$w_{5}(\alpha)$ & $1152+552 \alpha^{2}+94 \alpha^{4}+6 \alpha^{6}+\sqrt{\alpha^{2}+4}\left(576+204 \alpha^{4}+24 \alpha^{4}\right)$ \\
\hline$w_{6}(\alpha)$ & $216 \alpha^{2}+74 \alpha^{4}+6 \alpha^{6}+\sqrt{\alpha^{2}+4}\left(108 \alpha^{2}+24 \alpha^{4}\right)$ \\
\hline$w_{7}(\alpha)$ & $-5184-1824 \alpha^{2}-1128 \alpha^{4}-24 \alpha^{6}-\sqrt{\alpha^{2}+4}\left(2592+888 \alpha^{2}+72 \alpha^{4}\right)$ \\
\hline$w_{8}(\alpha)$ & $-\left(216+126 \alpha^{2}+18 \alpha^{4}\right) \sqrt{\alpha^{2}+4}$ \\
\hline$r_{1}(\alpha)$ & $22 \alpha^{2}+2 \alpha^{4}+4 \alpha^{2} \sqrt{\alpha^{2}+4}$ \\
\hline$r_{2}(\alpha)$ & $80+22 \alpha^{2}+2 \alpha^{4}+\left(32+4 \alpha^{2}\right) \sqrt{\alpha^{2}+4}$ \\
\hline$r_{3}(\alpha)$ & $-80-14 \alpha^{2}-2 \alpha^{4}-\left(32+4 \alpha^{2}\right) \sqrt{\alpha^{2}+4}$ \\
\hline$r_{4}(\alpha)$ & $-14 \alpha^{2}-2 \alpha^{4}-4 \alpha^{2} \sqrt{\alpha^{2}+4}$ \\
\hline$r_{5}(\alpha)$ & $32 \alpha+8 \alpha \sqrt{\alpha^{2}+4}$ \\
\hline$r_{6}(\alpha)$ & $32 \alpha+8 \alpha \sqrt{\alpha^{2}+4}$ \\
\hline
\end{tabular}

Table 3: We report the coefficients appearing in the expression of the Riemann, Weyl and Ricci invariants given, respectively, in Eqs. (B.1), (B.2) and (B.3). 


\section{References}

[1] G. Veneziano, Phys. Lett. B 265, 287 (1991).

[2] M. Gasperini and M. Giovannini, Phys. Rev. D 47, 1519 (1993); M. Gasperini, M. Giovannini and G. Veneziano Phys. Rev. Lett. 75, 3796 (1995); R. Brustein, M. Gasperini, M. Giovannini and G. Veneziano, Phys. Lett. B 361, 45 (1995); M. Giovannini, Phys. Rev. D 56, 3198 (1997); R. Brustein and M. Hadad, Phys. Rev. D 57, 725 (1998).

[3] C. Lovelace, Phys. Lett. B 135, 75 (1984); E.S. Fradkin and A. A. Tseytlin, Nucl. Phys. B 261, 1 (1985); C.G. Callan et al., Nucl. Phys. B 261, 593 (1985); A. Sen, Phys. Rev. Lett. $55,1846(1985)$.

[4] J. Barrow and K. Kunze, Phys. Rev. D 55, 623 (1997); ibid. 56, 741 (1997).

[5] G. Veneziano, Phys.Lett. B 406, 297 (1997).

[6] T. Piran and R. M. Williams, Phys. Lett. B 163, 331 (1985); H. Kurki-Suonio, J. Centerella, R. Matzner and J. Wilson, Phys. Rev. D 35, 453 (1987); D. Goldwirth and T. Piran, ibid. 40, 3263 (1989); ibid. 43, 3204 (1991); Phys. Rev. Lett. 64, 2852 (1990).

[7] M. Gasperini and G. Veneziano, Astropart. Phys. 1, 317 (1993).

[8] M. Turner and E. Weinberg, Phys. Rev. D 56, 4604 (1997); N. Kaloper, A. Linde and R. Bousso, Pre-big-bang requires the universe to be exponentially large from the vey beginning, hep-th/9801073.

[9] R. Brustein and G. Veneziano, Phys. Lett. B 329, 429 (1994); N. Kaloper, R. Madden and K. A. Olive, Nucl. Phys. B 452, 677 (1995); Phys. Lett. B 371, 34 (1996).

[10] I. Antoniadis, J. Rizos and K. Tamwakis, Nucl. Phys. B 415, 497 (1993); R. Easther and K. Maeda, Phys. Rev. D 54, 7252 (1996);R. Easther K. Maeda and D. Wands, Phys. Rev. D 53, 4247 (1996); S. J. Rey, Phys. Rev. Lett 77. 1929 (1996); M. Gasperini and G. Veneziano, Phys. Lett. B 387, 715 (1996); M. Gasperini, M. Maggiore and G. Veneziano, Nucl. Phys. B 494, 315 (1997); M. Gasperini and M. Giovannini Phys. Lett. B 287, 56 (1992). 
[11] E. J. Copeland, A. Lahiri and D. Wands, Phys. Rev. D 50, 4868 (1994); J. Levin, ibid. 51, 462 (1995); 51, 1536 (1995); E. J. Copeland, A. Lahiri and D. Wands, ibid. 51, 1569(1995); J. Levin, Phys. Lett. B 343, 69 (1995); J. E. Lidesy, ibid. 55, 3303 (1997); A. Lukas, B. A. Ovrut and D. Waldram, Nucl. Phys. B 495,365 (1997); R. Brustein and R. Madden, Phys. Rev. D 57, 712 (1998).

[12] M. Gasperini, J. Maharana and G. Veneziano, Phys. Lett. B 296, 51 (1997)

[13] A. Sen, Phys. Lett. B 271, 295 (1991); S. hassan and A. Sen, Nucl. Phys. B 375, 103 (1992).

[14] K. A. Meissner and G. Veneziano, Phys. Lett. B 267, 33 (1997); Mod. Phys. Lett. A 6, 3397 (1991); M. Gasperini, J. Maharana and G. Veneziano, Phys. Lett. B 272, 277 (1991); M. Gasperini and G. Veneziano, Phys. Lett. B 277, 256 (1992); K. A. Meissner ibid. 392, 298 (1997); N. Kaloper and K. Meissner, Phys. Rev. D 56, 7940 (1997).

[15] C. R. Nappi and E. Witten, Phys.Lett. B 293, 309 (1992).

[16] R. H. Brandenberger and C. Vafa, Nucl. Phys. B 316, 391 (1989); A. A. Tseytlin and C. vafa, Nucl. Phys. B 372, 443 (1992).

[17] A. Krasinski, Inhomogeneous Cosmological Models (Cambridge University Press, Cambridge 1997).

[18] M. Carmeli, Ch. Charach and S. Malin, Phys. Rep. 76, 80 (1981); J. Wainwright, W. C. Ince and B. J. Marshman, Gen. Relativ. Gravit. 10, 259 (1979).

[19] J. Wainwright, J. Phys. A 14, 1131 (1981); C. G. Hewitt and J. Wainwright, Class. Quantum Grav. 7,2295 (1990).

[20] E. Verdaguer, Phys. Rep. 229, 1 (1993).

[21] I. S. Gradshteyn and I. M. Ryzhik, Tables of Integrals, Series and Products, pag. 529 (Academic Press, Fifth edition, 1994).

[22] E. P. Liang, Astrophys. J. 204, 235 (1976); J. Wainwright and S. W, Goode, Phys. Rev. D 22, 1906 (1980). 
[23] M. Gasperini and G. Veneziano, Mod. Phys. Lett. A 8, 3701 (1993).

[24] N. Sanchez and G. Veneziano, Nucl.Phys. B 333, 253 (1990).

[25] S. W. Hawking and R. Penrose, Proc. R. Soc. London A314, 529 (1970); S. W. Hawking and G. F. R. Ellis, The Large Scale Structure of the Universe (Cambridge Univ. Press, Cambridge 1973).

[26] R. M. Wald, General Relativity, (Chicago University Press, Chicago 1984).

[27] F. J. Chinea, L. Fernandez-Jambrina and J. M. Senovilla, Phys. Rev. D 45, 481 (1992). 